\title{
Addition of shock wave therapy to nail dynamization increases the chance of long-bone non-union healing
}

\author{
Josef Stolberg-Stolberg ${ }^{1}$, Thomas Fuchs², Moritz F. Lodde' , Steffen Roßlenbroich', Patric Garcia', \\ Michael Raschke ${ }^{1 *}$ and Jens Everding ${ }^{1}$
}

\begin{abstract}
Background: Long-bone non-unions after intramedullary nailing can be treated by nail dynamization or focused high-energy extracorporal shock wave therapy (fESWT). The objective of this study was to assess the effect of the combination therapy of nail dynamization and fESWT on long-bone non-unions.
\end{abstract}

Materials and methods: 49 patients with long-bone non-unions (femur and tibia) after nailing were treated with nail dynamization (group $D, n=15$ ), fESWT (group $S, n=17$ ) or nail dynamization in addition to fESWT (group DS, $n=17)$. Patients were followed up for 6 months retrospectively. Furthermore, age, sex, Non-Union Scoring System (NUSS) score, time intervals from primary and last surgery until intervention and smoking status were analysed for their correlations to bone union.

Results: Union rates were 60\% for group D, 64.7\% for group S and 88.2\% for group DS, with a significant difference between group D and DS ( $p=0.024)$. Successful treatment was correlated with high age (OR $1.131 ; 95 \% \mathrm{Cl}$ $1.009-1.268 ; p=0.034)$, female gender (OR 0.009; $95 \% \mathrm{Cl} 0.000-0.89 ; p=0.039$ ), low NUSS score (OR $0.839 ; 95 \% \mathrm{Cl}$ $0.717-0.081 ; p=0.028$ ) and negative smoking status (OR 86.018;95\% Cl 3.051-2425.038; $p=0.009$ ).

Conclusions: Data from the present study indicate that the combination therapy of nail dynamization and fESWT leads to a higher union rate than dynamization or fESWT alone.

Level of evidence: Level 3.

Keywords: Shock wave, Non-union, Nail dynamization, Long bones

\section{Introduction}

Fracture healing is a complex regenerative process that usually results in scar-free bone union [1]. However, $1.9-10 \%$ of all fractures show impaired healing, with the lower leg being at particular risk: non-union rates of up to $50 \%$ are observed in cases of open fracture [24]. While a significant effort has been made to improve

\footnotetext{
*Correspondence: Michael.Raschke@ukmuenster.de

1 Department of Trauma, Hand and Reconstructive Surgery, University Hospital Muenster, Albert-Schweitzer-Campus 1, Building W1, 48149 Muenster, Germany

Full list of author information is available at the end of the article
}

non-union treatment, the underlying processes are not well understood. Hence, the diamond concept provides a structural framework that emphasizes the importance of biological and mechanical factors in osseous union [5].

One of the most commonly performed procedures to mechanically accelerate fracture healing after nailing of long bones is nail dynamization. This comprises the removal of proximal or distal locking screws in statically locked intramedullary nails in order to reduce the fracture gap and increase the compression force. This technique was initially intended to improve union rates during normal fracture healing. It is an economical and technically simple procedure for the treatment of 
long-bone non-unions. However, clinical studies are yet to prove a beneficial effect [6]; reported union rates range between 30 and $90 \%$, and clinical studies are difficult to compare [7-12]. Factors affecting treatment success include time since fracture and the callus-to-diaphysis ratio $[13,14]$. Meta-analyses and systematic reviews are scarce [6].

For clinical use, the Moghaddam risk score has been developed as a means to estimate the risk for non-union formation [15]. Furthermore, the Non-Union Scoring System (NUSS) assigns specialized treatment strategies to non-unions according to their NUSS scores. Factors such as open fracture, clinical infection signs and smoking status have been identified as favouring non-union formation. The Ladder Strategy recommends the standard treatment for patients with scores of $0-25$, a specialized treatment such as fESWT, growth factors or mesenchymal stromal cells for patients with between 26 and 50 points, bone resection and transport as well as microvascular flaps for patients with $51-75$ points, and arthrodesis, mega-prosthesis or even amputation in patients with scores above 76 points $[15,16]$. The aim of the present study was to investigate the effect of additional shock wave treatment on nail dynamization of long-bone non-unions.

High-energy focused extracorporal shock wave therapy (fESWT) is a non-invasive outpatient procedure to biologically stimulate fracture healing in non-unions [17]. Shock waves are single acoustic impulses with a rapid pressure increase followed by a tensile phase with a low amplitude generated by an electrohydraulic, electromagnetic or piezoelectric mechanism [18]. Cell and extracellular matrix stimulation induce the expression of angiogenetic and osteogenetic factors that are important for bone healing [19]. Exclusion of infection and achievement of the correct bone alignment, length, rotation and non-union gap $(<5 \mathrm{~mm})$ are reported to be required for successful fESWT application. Clinical studies show union rates of between 60 and $87 \%$ and state that fESWT and revision surgery are equally effective [20-22]. However, fESWT is not widely used, and revision surgery including non-union resection, bone grafting and re-fixation remains the gold standard for most clinics and most non-unions.

Until the present work, no study had compared fESWT and nail dynamization or analysed the effect of combining fESWT and nail dynamization on long-bone nonunions. fESWT can also be applied in cases of non-union where nail dynamization is used to further stimulate bone healing. This can be done without incurring significant financial expense, increasing the surgery time or elevating the risk for complications. The aim of this study was to analyse the effect of adding fESWT to nail dynamization in diaphyseal non-union treatment, and to show its feasibility in clinical practice.

\section{Materials and methods}

This study was conducted as a retrospective, monocentric study during 2014-2019. It was authorized by the local ethics committee (the ethics committee of the Medical Association of Westfalen-Lippe, no: 2016-160$\mathrm{f}-\mathrm{S}$. Bone healing was defined as the presence of at least tricortical bridging callus and painless full weight bearing 6 months after trauma.

\section{Inclusion criteria}

The clinical information system was scanned for the search terms "shock wave" and "dynamization". All cases were viewed manually, and only patients with long-bone non-unions (non-union gap $\leq 5 \mathrm{~mm}$ ) after nailing of the femur or tibia who received only shock wave treatment (group S), only nail dynamization (group D) or the combination of both (group DS) were included. Patient history was scanned for signs of infection at the site of treatment and patients with elevated infectious blood parameters were excluded. Radiographic images taken at the time of treatment were examined and patients with implant loosening, axial deviation or signs of procedural implant errors were not included. All patients with additional procedures such as autologous bone grafting or bone morphogenetic protein application were excluded (Fig. 1).

Patient assignment to groups D, S, and DS was performed based on the decision of the treating senior physician. All patients agreed by written consent to the treatment procedure. Additionally, patient age, sex, AOMueller classification, anatomic region, size of the nonunion gap, time between trauma and treatment, time since last surgery, and NUSS score were assessed at treatment (Table 1) [16].

\section{High-energy focused extracorporal shock wave treatment and dynamization}

All patients were treated under either general or regional anaesthesia. An electrohydraulic shock wave device (Lithospace Ortho ${ }^{\circledR}$, JenaMedtech) was used for all shock wave therapies. Non-union was visualized by $\mathrm{X}$-ray and the level was marked on the skin. Anatomical structures such as arteries, veins and nerves were spared by the shock waves. The shock wave depth (therapeutic volume at $-6 \mathrm{~dB}: 16.6 \mathrm{~mm} \times 104.2 \mathrm{~mm}$ ) was adjusted to the soft tissue thickness. In total, $3 \times 1000$ impulses at $23 \mathrm{kV}$ were applied with an energy flux density of $0.36 \mathrm{~mJ} / \mathrm{mm}^{2}$ at $4 \mathrm{~Hz}$ from different angles. Ultrasonic gel allowed energy transmission. Nail dynamization was done using a sterile technique and 


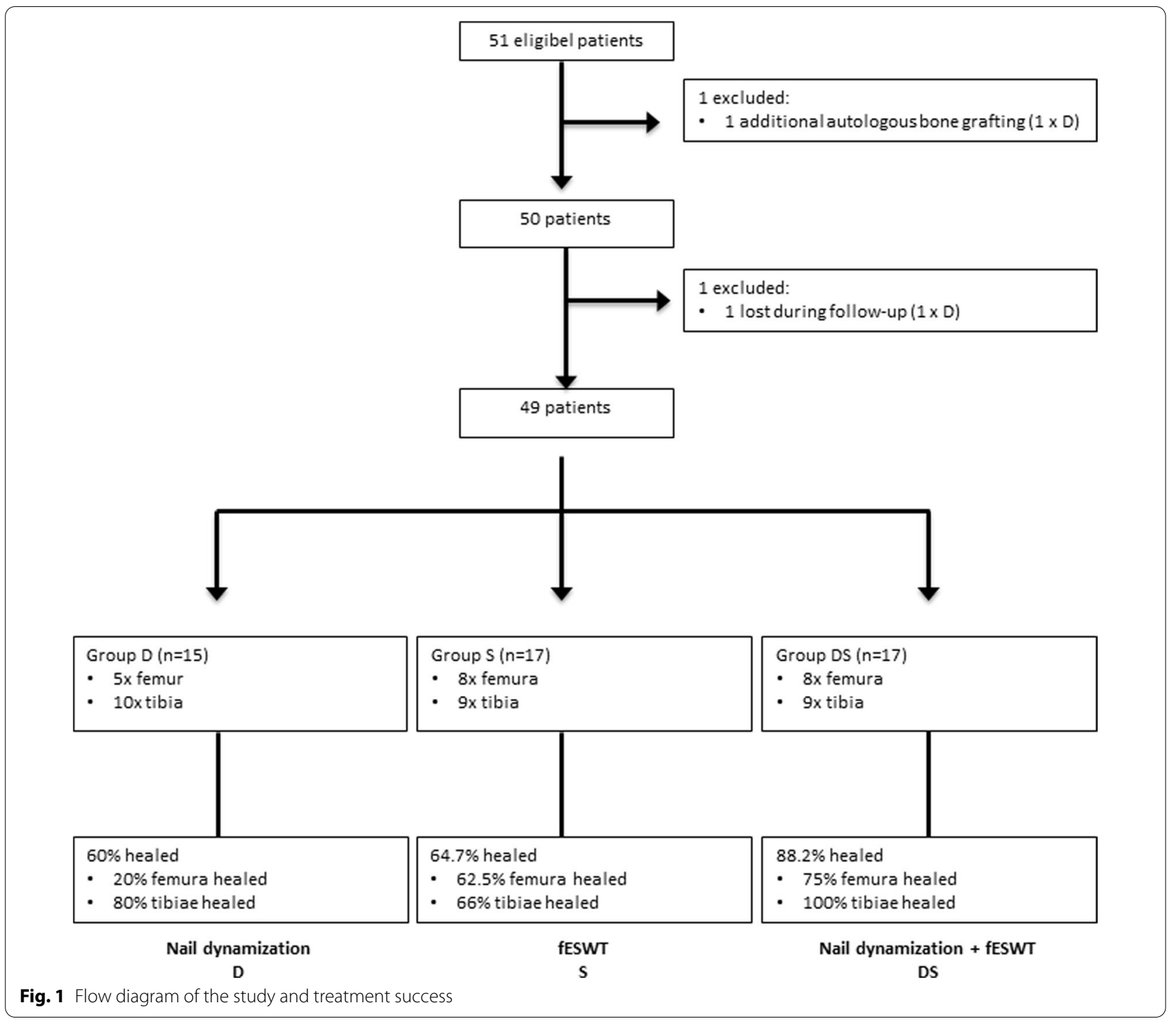

tools provided by the manufacturer. All locking screws were removed with a single skin incision, careful softtissue preparation and without screw breakage. Wound suture and a sterile wound dressing were applied.

Rare complications such as petechiae, neurovascular deficit or excessive pain were not found in any patient shortly after the fESWT intervention. Groups S and DS were allowed partial weight bearing for 6 weeks using crutches and thrombosis prophylaxis, while group D started full weight bearing directly after dynamization. All patients were followed up for 6 months. Non-union healing was assessed 6 months after treatment by CT scan or two plain X-rays and clinical examination by two senior orthopaedic surgeons (Fig. 2).

\section{Statistics}

Statistical analyses were performed using IBM SPSS ${ }^{\circledR}$ Statistics 26 (IBM Corporation, Somers, NY, USA). Oneway ANOVA, Kruskal-Wallis and Fischer's exact test were performed to account for group differences. Logistic regression was conducted to analyse risk-adjusted odds ratios.

\section{Results}

\section{Demographics}

In total, 49 patients were followed up for 6 months during the years 2014-2019. Only long-bone shaft nonunions of the femur (AO 32) or tibia (AO 42) treated with intramedullary nailing were included in this 


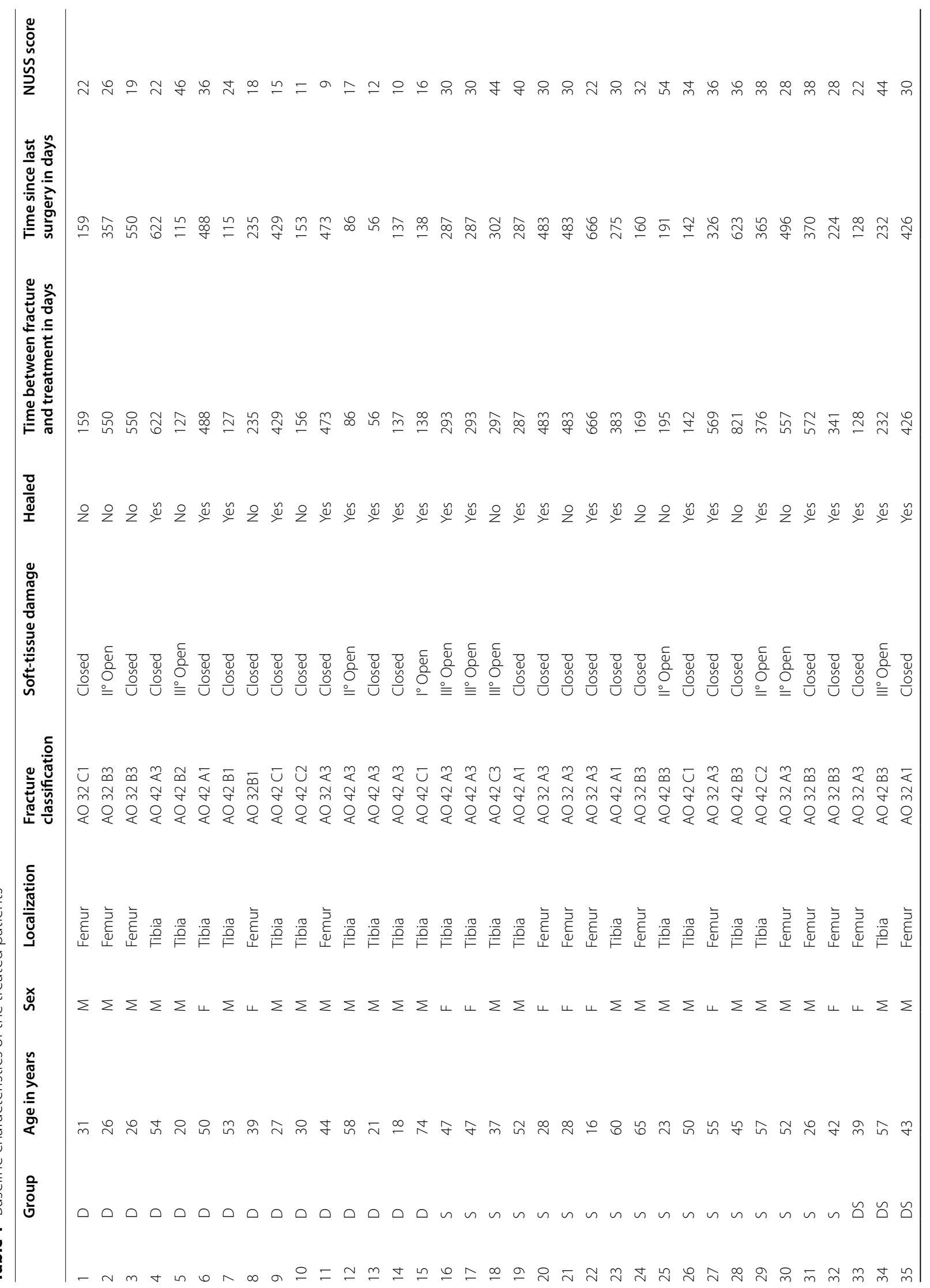




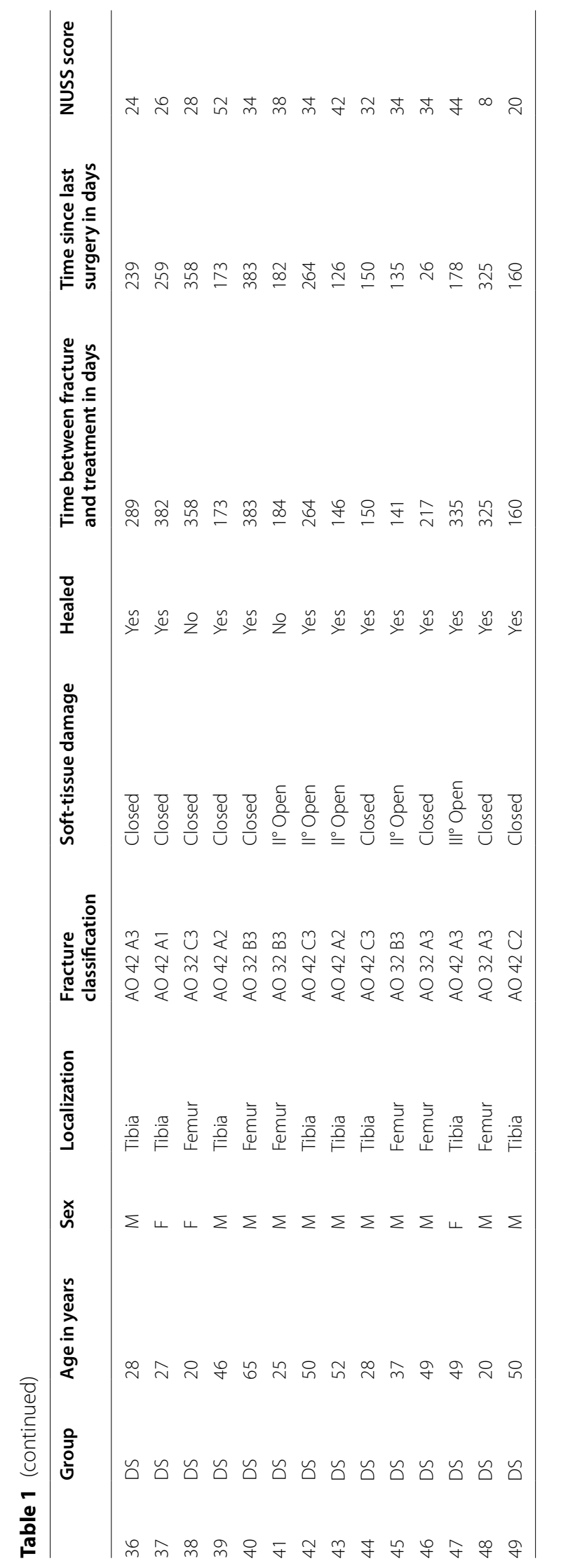



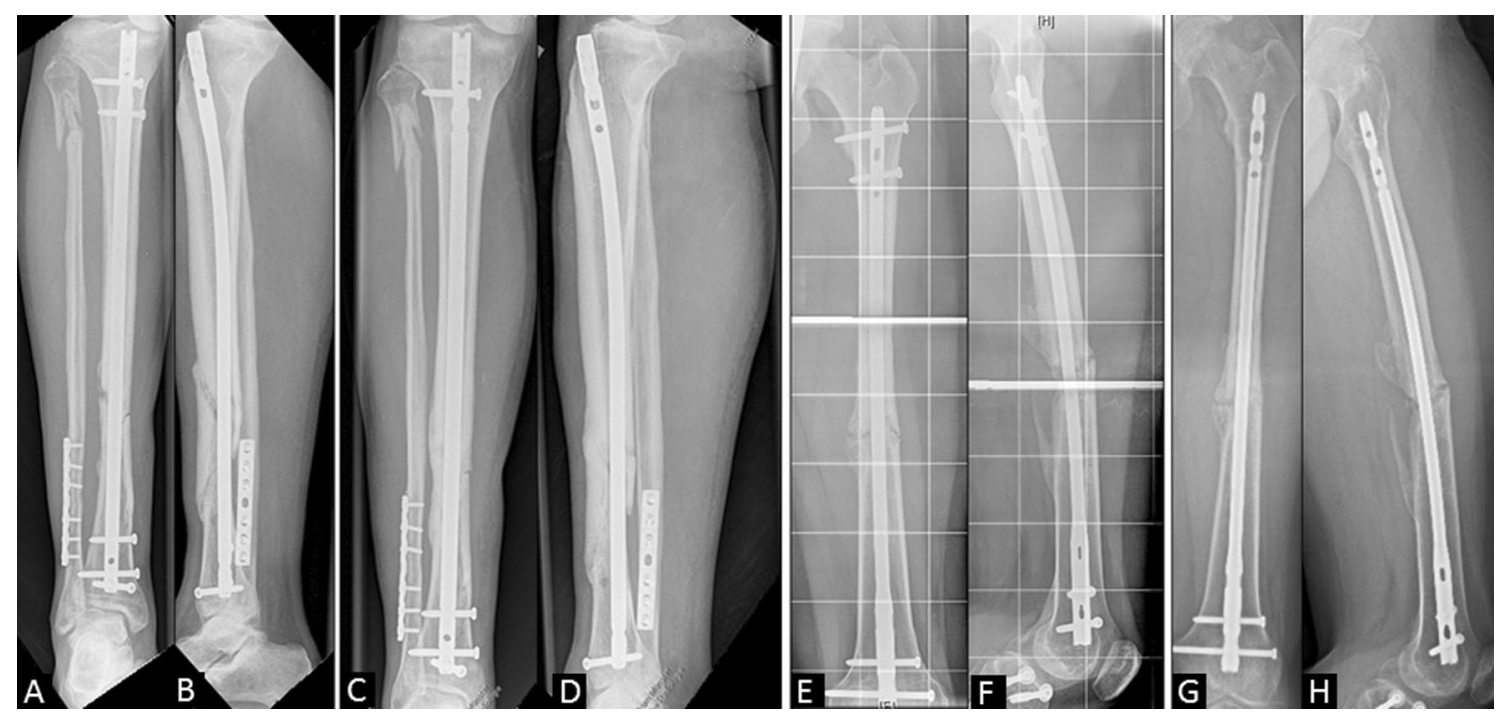

Fig. 2 A-D Serial radiographs of a 50-year-old patient after a $I^{\circ}$ open tibia fracture showing non-union 8 months after primary surgery $($ A, B) and osseous union 6 months after nail dynamization and fESWT (C, D). E-G A 27-year-old patient suffered from a closed femur fracture with consecutive non-union 1.5 years later $(\mathbf{E}, \mathbf{F})$. Treatment of the clinical control with nail dynamization was not successful after 6 months $(\mathbf{G}, \mathbf{H})$

study. Fractures and soft-tissue injuries were classified according to the AO-Mueller and Gustilo-Anderson classifications (Table 1) [23]. Altogether, group D consisted of 15 patients $(5 \times$ femur, $10 \times$ tibia), group $\mathrm{S}$ consisted of 17 patients $(8 \times$ femur, $9 \times$ tibia $)$ and group DS consisted of 17 patients $(8 \times$ femur, $9 \times$ tibia $)$. The arithmetic mean ages of the 13 female and 36 male patients were $41.6( \pm 13.1)$ and $40.6( \pm 14.65)$ years, respectively. There was no difference in age or sex between groups $(p>0.05)$. The time interval between primary injury and non-union treatment using dynamization and/or fESWT was 289 ( \pm 202 ) days for group D, 407 ( \pm 186.4) days for group $S$ and 252.5 ( \pm 100 ) days for group DS, with no significant differences between groups (Table 1). There was an average time of 275 $( \pm 191)$ days, $351( \pm 153)$ days and 220 ( \pm 106$)$ days for groups D, S and DS, respectively, between the last surgery and non-union treatment. Group $\mathrm{S}$ was treated significantly earlier than group DS $(p=0.045)$. Patients in group D had a significantly lower NUSS score of $19.5( \pm 11)$ than those in groups S and DS: $34( \pm 7.4)$ and $37.9( \pm 10.3)$, respectively $(p<0.006)$. Group D had an average non-union gap of $2.2( \pm 0.42) \mathrm{mm}$, that for group $\mathrm{S}$ was $2.9( \pm 1.1) \mathrm{mm}$ and that for group DS was $2.8( \pm 0.72) \mathrm{mm}$, with the non-union gap in group $\mathrm{D}$ being significantly smaller than that in group DS $(p=0.029)$. There were no significant differences regarding smoking, soft-tissue damage or type of nonunion between groups.

\section{Primary outcome measures}

Non-union healing was assessed 6 months after intervention, and significant differences between groups were observed $(p=0.045)$. Groups D, S and DS showed union rates of $60 \%, 64.7 \%$ and $88.2 \%$. There was no significant difference between groups $\mathrm{D}$ and $\mathrm{S}$ according to a riskadjusted comparison $(p=0.65)$. However, the union rate of group DS was significantly higher than that of group D $(p=0.024)$ (Fig. 3).

\section{Secondary outcome measures}

Taking all patients together, age (OR 1.131; 95\% CI $1.009-1.268 ; p=0.034)$ and sex (OR $0.009 ; 95 \%$ CI $0.000-0.89 ; p=0.039)$ had significant effects on nonunion healing, with older and female patients showing higher probabilities of bone union (Fig. 4). Increasing NUSS score was linked to a decreasing chance of nonunion healing (OR 0.839; 95\% CI 0.717-0.081; $p=0.028$ ). Furthermore, smoking reduced the probability of bone union (OR 86.018; 95\% CI 3.051-2425.038; $p=0.009$ ). Time interval between accident and intervention, time interval between last surgery and intervention, type of non-union (hypertrophic, atrophic) and soft-tissue damage (open, closed) had no effect on treatment success.

\section{Discussion}

The objective of this study was to investigate whether high-energy shock wave therapy in addition to nail dynamization improves bone healing after long-bone 


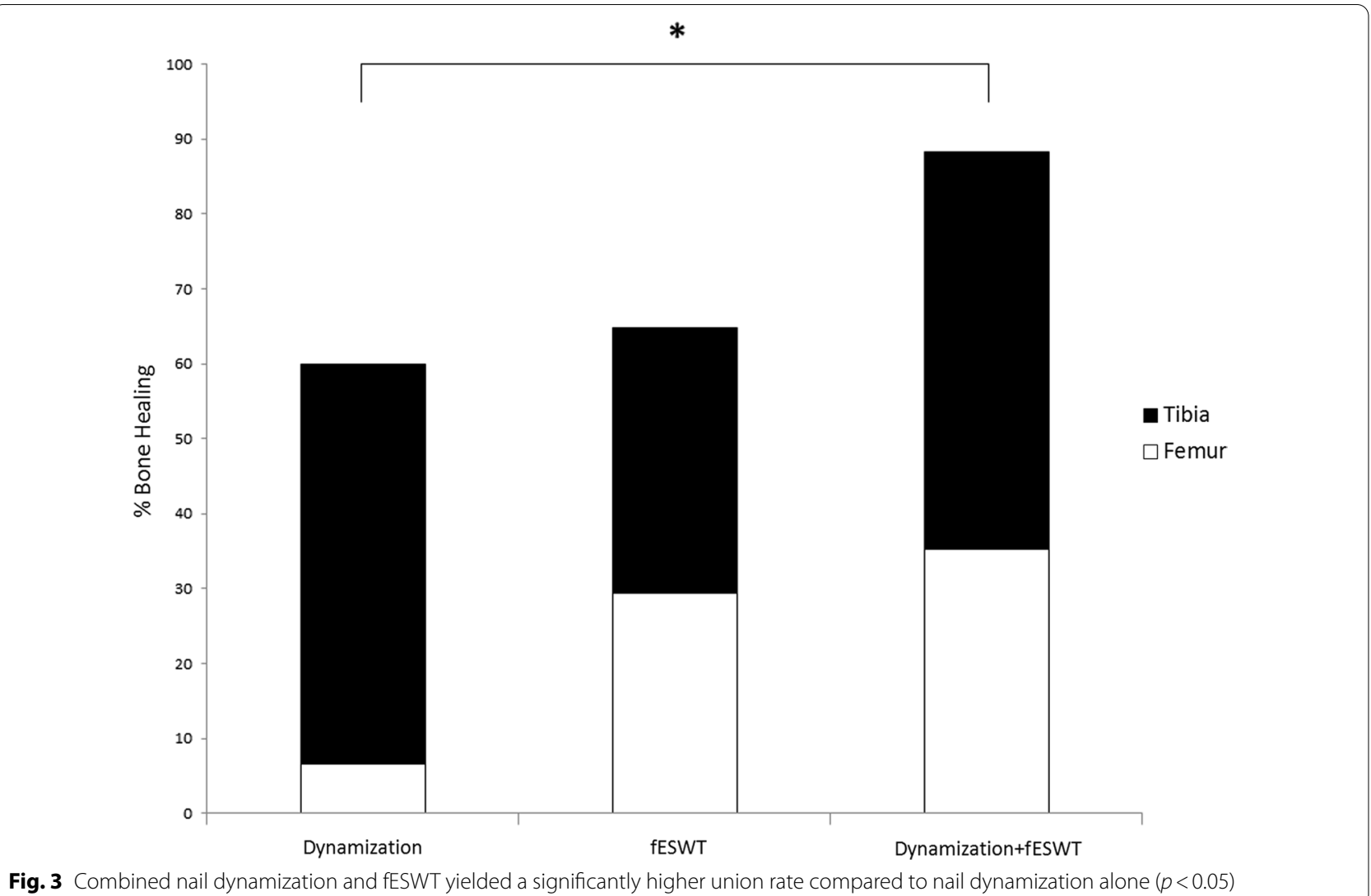

Fig. 3 Combined nail dynamization and fESWT yielded a significantly higher union rate compared to nail dynamization alone $(p<0.05)$

\section{Increasing chances of non-union healing}

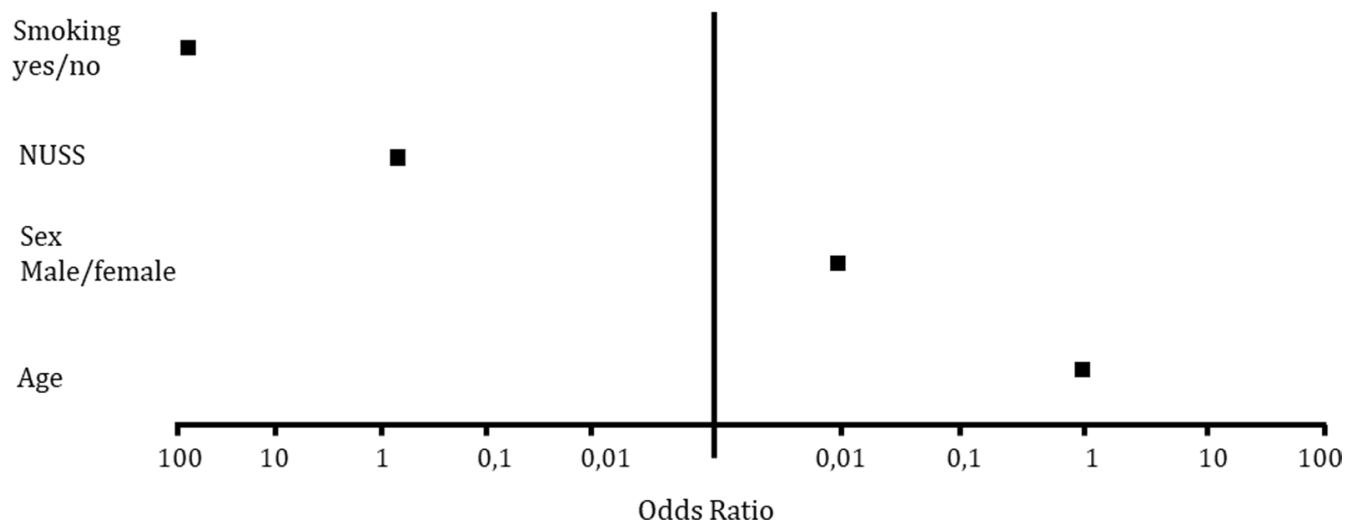

Fig. 4 Odds ratios for bone union according to smoking status (yes/no), NUSS score, sex (male/female) and age

non-union. The diamond concept, a conceptual framework for non-union treatment, suggests that four factors-ososteogenic cells, mechanical stability, an osteoconductive matrix and osteoinductive mediators-are all essential for non-union healing. However, no study has investigated the combination of fESWT (for biological stimulation) with nail dynamization (to mechanically alter stability) to improve fracture healing $[5,24]$.

Originally, nail dynamization of long bones was performed 10-16 weeks after nail implantation to promote further callus modelling at the fracture site [25-27]. Recent literature, however, does not consider it mandatory, and even emphasizes harmful side effects such as 
limb shortening and prolonged treatment duration [10, 11, 28]. Furthermore, in vivo data on sheep and dogs with comminuted tibia fractures or unstable osteotomies did not demonstrate a beneficial effect of dynamization during normal fracture healing [29-31]. Subsequently, nail dynamization has become a specific procedure for use in cases of non-union, although the literature shows great variation in union rates $[6,32]$. Vaughn et al. analysed 35 patients with dynamization of femoral and tibial non-unions. A 54\% healing rate and a correlation between callus diameter at the time of dynamization and union rate was found [14]. Litrenta et al. reported an $83 \%$ tibial union rate by nail dynamization after a median of 156 days, and emphasized that a fracture gap of $>5 \mathrm{~mm}$ was a statistically negative factor for bone union [33]. The results of the present study show a union rate of $60 \%$ after dynamization only. In contrast to Vincenti et al., we did not observe a correlation between time from fracture until dynamization and union rate [13]. This emphasizes the need for combination treatment modalities such as nail dynamization and biophysical stimulation to further increase rates of non-union healing.

fESWT is a minimally invasive treatment option for delayed unions and non-unions [34]. Constant osseous healing rates of between 60 and $80 \%$ are found in the literature $[18,35,36]$. The $65 \%$ union rate observed in the present study after 6 months of follow-up is lower than that reported in an earlier publication from our group [18]. This might be explained by the exclusive selection of patients with intramedullary nail stabilization at the femur and tibia only in the present work, as tibia and femur fractures have been reported to have impaired outcomes compared to other bones. This is consistent with findings by Stojadinovic et al., who analysed 349 cases of delayed fracture healing and non-unions to develop a prognostic naïve Bayesian classifier model and identified the femur as well as intramedullary stabilization as negative healing predictors [37]. Furthermore, most previous studies did not analyse the NUSS score, which might also explain differences in union rate due to the nature of the specific non-union.

Regarding non-union treatment, only two studies have analyzed the effect of the combined application of treatment modalities. Cebriàn et al. included 57 cases of tibial non-union that were all treated surgically. In 22 of the cases, pulsed electromagmetic field (PMEF) therapy was also applied without achieving significantly increased healing rates [38]. Zhai et al. reported that significantly higher union rates of long-bone non-unions were obtained when applying autologous mesenchymal stem cells additionally to fESWT [39]. To the best knowledge of the authors, the present study describes the combination of fESWT, a biophysical procedure, with nail dynamization for the first time. Similar to PMEF, fESWT is a fast, inexpensive and low-risk procedure that can be easily performed during nail dynamization. The present study shows that bone union increased from $60 \%$ with dynamization alone to $88 \%$ with dynamization + fESWT. This result is made even more important by the fact that the DS group showed a significantly higher NUSS score than the D group. This shows that the combination of dynamization and fESWT significantly improves bone healing, even for more severe non-unions. The increased union rate after combined dynamization and fESWT could help to reduce treatment costs because of the reduced need for subsequent treatments and earlier social and occupational re-integration. This should more than compensate for the additional costs of fESWT during nail dynamization.

Female gender was correlated with an increased probability of bone union. Although there is no evidence in the literature that sex affects non-union healing, male patients tend to undertake higher-risk activities that can lead to high-energy fractures [40,41]. This is consistent with the present study, in which $34 \%$ of the fractures in male patients and $23 \%$ of those in female patients were open. Interestingly, older patients had a higher probability of non-union healing. While the influence of age on bone healing is a controversial subject in the literature, most authors state that age may be a surrogate for the prevalence of other risk factors, such as fracture location, soft-tissue damage, secondary diseases such as diabetes, and the use of NSAIDs $[40,41]$. The NUSS score provides a tool for comparing a heterogeneous group of patients with osseous non-unions [16, 42]. It is recommended that patients with a high NUSS score (26-50 points) should be considered for more specialized treatment, including biological stimulation with fESWT or PMEF. With their average NUSS score of 29.2 ( \pm 10.9 ), the patients in the present study meet the recommended inclusion criteria [43]. Additionally, patients with higher NUSS scores showed a decreased chance of bone union, which is consistent with the literature. As expected, smoking reduced the chance of non-union healing [44].

The follow-up treatment after fESWT is a controversial topic in the literature [45]. Most authors, such as those of the current study, recommend reduced weight bearing after fESWT in order to promote local biological processes such as angiogenesis and the release of inflammatory cytokines [46-48]. All patients in groups $\mathrm{S}$ and DS in the present study were therefore advised to adopt partial $(20 \mathrm{~kg})$ weight bearing on crutches for 6 weeks. Patients in group D were allowed full weight bearing in accordance with current recommendations for early or late dynamization of femoral or tibial shaft fractures. Axial compression due to full weight bearing 
after dynamization has been recognized to improve fracture healing $[49,50]$. According to those studies, we would expect improved fracture healing in the D group (full weight bearing) compared to the S and DS groups (20 kg weight bearing). Therefore, we have to conclude that either (i) current concepts of full weight bearing after dynamization are not accurate or (ii) the additional fESWT more than compensated for the effects of partial weight bearing, leading to a significantly higher union rate in the DS group. Further studies are needed to analyze the influence of weight bearing on a fracture after dynamization alone.

This retrospective study provides the first evidence of treatment success using the combination therapy of nail dynamization and fESWT. However, the study is limited by the number of patients (49), its non-randomized patient selection and its non-blinded and retrospective design. The NUSS score of group D was significantly lower than those of groups S and DS. Similarly, group D had a significantly smaller non-union gap compared to group DS. However, despite the lower NUSS score and smaller non-union gap in group D, the combination of nail dynamization and fESWT (group DS) resulted in a significantly higher union rate. Wide confidence intervals regarding the smoking status were observed, but there was still a statistically significant difference, with poorer outcomes for smokers. Strengths of the present study comprise the homogeneous cohort of patients with diaphyseal fractures of the femur and tibia only, the exclusion of infected non-unions, and correct implant positioning. All patients received the same primary operative treatment with intramedullary nailing.

In conclusion, the present study shows that implementation of fESWT additionally to nail dynamization significantly improves fracture healing in long-bone diaphyseal non-unions. The procedure is minimally invasive and can be performed as an outpatient procedure with excellent union rates if indicated and performed correctly. In comparison to the current gold standard of non-union treatment, which includes non-union resection, bone grafting and re-osteosynthesis, we present a cost-effective, lowrisk alternative treatment option. However, to confirm our results, future randomized prospective and blinded studies are necessary to create a higher degree of clinical evidence.

\section{Abbreviations}

fESWT: Focused high-energy extracorporal shock wave therapy; NUSS: NonUnion Scoring System; Group D: Group treated with nail dynamization; Group S: Group treated with fESWT; Group DS: Group treated with nail dynamization in addition to fESWT; PMEF: Pulsed electromagmetic fields.
Acknowledgements

We acknowledge support from the Open Access Publication Fund of the University of Muenster.

\section{Authors' contributions}

Conception and study design: JE, JS-S, PG, MR. Drafting of article: JS-S, JE, MR. Critical review of the article: all authors. Provision of study materials: $M R, M L, T F$ SR, JS-S, JE, PG. Data acquisition and analysis: JE, SR, JS-S, TF, ML, PG. All authors have read and approved the final manuscript.

\section{Funding}

There was no funding for this study.

\section{Availability of data and materials}

The datasets used and analysed during the current study are available from the corresponding author on reasonable request.

\section{Declarations}

Ethics approval and consent to participate

This study was authorized by the local ethics committee (the ethics committee of the Medical Association of Westfalen-Lippe, no: 2016-160-f-S).

\section{Consent of publication}

Not applicable. Due to the retrospective study design, the need for written consent to participate in the study was waived by the ethics committee. However, for the treatment itself, written consent was obtained from each patient.

\section{Competing interests}

The authors declare that they have no competing interests.

\section{Author details}

${ }^{1}$ Department of Trauma, Hand and Reconstructive Surgery, University Hospital Muenster, Albert-Schweitzer-Campus 1, Building W1, 48149 Muenster, Germany. ${ }^{2}$ Department of Trauma and Reconstructive Surgery, Vivantes Hospital Friedrichshain, Landsberger Allee 49, 10249 Berlin, Germany.

Received: 9 March 2021 Accepted: 26 September 2021

Published online: 08 January 2022

\section{References}

1. Einhorn TA, Gerstenfeld LC (2015) Fracture healing: mechanisms and interventions. Nat Rev Rheumatol 11:45-54. https://doi.org/10.1038/ nrrheum.2014.164

2. Ekegren CL, Edwards ER, de Steiger R, Gabbe BJ (2018) Incidence, costs and predictors of non-union, delayed union and mal-union following long bone fracture. Int J Environ Res Public Health 15:2845. https://doi. org/10.3390/ijerph15122845

3. Mills LA, Aitken SA, Simpson AHRW (2017) The risk of non-union per fracture: current myths and revised figures from a population of over 4 million adults. Acta Orthop 88:434-439. https://doi.org/10.1080/17453 674.2017.1321351

4. Zimmermann G, Wagner C, Schmeckenbecher K et al (2009) Treatment of tibial shaft non-unions: bone morphogenetic proteins versus autologous bone graft. Injury 40(Suppl 3):S50-S53. https://doi.org/10.1016/S00201383(09)70012-9

5. Andrzejowski P, Giannoudis PV (2019) The "diamond concept" for long bone non-union management. J Orthop Traumatol 20:1-13. https://doi. org/10.1186/s10195-019-0528-0

6. Vaughn JE, Shah RV, Samman T et al (2018) Systematic review of dynamization vs exchange nailing for delayed/non-union femoral fractures. World J Orthop 9:92-99. https://doi.org/10.5312/wjo.v9.i7.92

7. Huang K-C, Tong K-M, Lin Y-M et al (2012) Evaluation of methods and timing in nail dynamisation for treating delayed healing femoral shaft fractures. Injury 43:1747-1752. https://doi.org/10.1016/j.injury.2012.06. 024

8. Papakostidis C, Psyllakis I, Vardakas D et al (2011) Femoral-shaft fractures and nonunions treated with intramedullary nails: the role of 
dynamisation. Injury 42:1353-1361. https://doi.org/10.1016/j.injury.2011. 06.024

9. Pihlajamäki HK, Salminen ST, Böstman OM (2002) The treatment of nonunions following intramedullary nailing of femoral shaft fractures. J Orthop Trauma 16:394-402. https://doi.org/10.1097/00005131-20020 7000-00005

10. Wu CC, Chen WJ (1997) Healing of 56 segmental femoral shaft fractures after locked nailing. Poor results of dynamization. Acta Orthop Scand 68:537-540. https://doi.org/10.3109/17453679708999022

11. Wu CC (1997) The effect of dynamization on slowing the healing of femur shaft fractures after interlocking nailing. J Trauma 43:263-267. https://doi. org/10.1097/00005373-199708000-00010

12. Wu CC, Shih CH (1993) Effect of dynamization of a static interlocking nail on fracture healing. Can J Surg 36:302-306

13. Vicenti G, Bizzoca D, Carrozzo M et al (2019) The ideal timing for nail dynamization in femoral shaft delayed union and non-union. Int Orthop 43:217-222. https://doi.org/10.1007/s00264-018-4129-y

14. Vaughn JE, Gotha $\mathrm{H}$, Cohen $\mathrm{E}$ et al (2016) Nail dynamization for delayed union and nonunion in femur and tibia fractures. Orthopedics 39:e1117e1123. https://doi.org/10.3928/01477447-20160819-01

15. Moghaddam A, Zimmermann G, Hammer K et al (2011) Cigarette smoking influences the clinical and occupational outcome of patients with tibial shaft fractures. Injury 42:1435-1442. https://doi.org/10.1016/j.injury. 2011.05.011

16. Calori GM, Phillips M, Jeetle S et al (2008) Classification of non-union: need for a new scoring system? Injury 39(Suppl 2):S59-63. https://doi.org/ 10.1016/S0020-1383(08)70016-0

17. Georg Thieme Verlag KG, Everding J, Stolberg-Stolberg J et al (2019) Möglichkeiten der nicht operativen Behandlung von Pseudarthrosen. OP J 35:252-261. https://doi.org/10.1055/a-0899-0068

18. Everding J, Freistühler M, Stolberg-Stolberg JA et al (2017) Extracorporal shock wave therapy for the treatment of pseudarthrosis: new experiences with an old technology. Unfallchirurg 120:969-978. https://doi.org/ 10.1007/s00113-016-0238-5

19. Wang C-J, Wang F-S, Yang KD (2008) Biological effects of extracorporeal shockwave in bone healing: a study in rabbits. Arch Orthop Trauma Surg 128:879-884. https://doi.org/10.1007/s00402-008-0663-1

20. Cacchio A, Giordano L, Colafarina O et al (2009) Extracorporeal shockwave therapy compared with surgery for hypertrophic long-bone nonunions. J Bone Joint Surg Am 91:2589-2597. https://doi.org/10.2106/ JBJS.H.00841

21. Notarnicola A, Moretti L, Tafuri S et al (2010) Extracorporeal shockwaves versus surgery in the treatment of pseudoarthrosis of the carpal scaphoid. Ultrasound Med Biol 36:1306-1313. https://doi.org/10.1016/j.ultra smedbio.2010.05.004

22. Furia JP, Juliano PJ, Wade AM et al (2010) Shock wave therapy compared with intramedullary screw fixation for nonunion of proximal fifth metatarsal metaphyseal-diaphyseal fractures. J Bone Joint Surg Am 92:846-854. https://doi.org/10.2106/JBJS.I.00653

23. Gustilo RB, Anderson JT (1976) Prevention of infection in the treatment of one thousand and twenty-five open fractures of long bones: retrospective and prospective analyses. J Bone Joint Surg Am 58:453-458

24. Giannoudis PV, Einhorn TA, Marsh D (2007) Fracture healing: the diamond concept. Injury 38(Suppl 4):S3-6

25. Kempf I, Grosse A, Beck G (1985) Closed locked intramedullary nailing. Its application to comminuted fractures of the femur. J Bone Joint Surg Am 67:709-720

26. Winquist RA, Hansen ST (1980) Comminuted fractures of the femoral shaft treated by intramedullary nailing. Orthop Clin North Am 11:633-648

27. Rosa N, Marta M, Vaz M et al (2019) Intramedullary nailing biomechanics: evolution and challenges. Proc Inst Mech Eng H 233:295-308. https://doi. org/10.1177/0954411919827044

28. Tigani D, Fravisini M, Stagni C et al (2005) Interlocking nail for femoral shaft fractures: is dynamization always necessary? Int Orthop 29:101-104. https://doi.org/10.1007/s00264-004-0627-1

29. Dagrenat D, Moncade N, Cordey J et al (1988) Effects of the dynamization of static bolt nailing. In vivo experimentation. Rev Chir Orthop Reparatrice Appar Mot 74(Suppl 2):100-104

30. Durall I, Falcón C, Díaz-Bertrana MC, Franch J (2004) Effects of static fixation and dynamization after interlocking femoral nailing locked with an external fixator: an experimental study in dogs. Vet Surg 33:323-332. https://doi.org/10.1111/j.1532-950X.2004.04047.x

31. Georgiadis GM, Minster GJ, Moed BR (1990) Effects of dynamization after interlocking tibial nailing: an experimental study in dogs. J Orthop Trauma 4:323-330. https://doi.org/10.1097/00005131-199004030-00015

32. Glatt V, Evans CH, Tetsworth K (2016) A concert between biology and biomechanics: the influence of the mechanical environment on bone healing. Front Physiol 7:678. https://doi.org/10.3389/fphys.2016.00678

33. Litrenta J, Tornetta P, Vallier $\mathrm{H}$ et al (2015) Dynamizations and exchanges: success rates and indications. J Orthop Trauma 29:569-573. https://doi. org/10.1097/BOT.0000000000000311

34. Everding J, Stolberg-Stolberg JA, Raschke MJ, Stange R (2019) Stimulation of fracture healing by growth factors and cell-based technologies. Unfallchirurg 122:534-543. https://doi.org/10.1007/s00113-019-0686-9

35. Elster EA, Stojadinovic A, Forsberg J et al (2010) Extracorporeal shock wave therapy for nonunion of the tibia. J Orthop Trauma 24:133-141. https://doi.org/10.1097/BOT.0b013e3181b26470

36. Wang C-J, Chen HS, Chen CE, Yang KD (2001) Treatment of nonunions of long bone fractures with shock waves. Clin Orthop Relat Res 387:95-101

37. Stojadinovic A, Kyle Potter B, Eberhardt J et al (2011) Development of a prognostic naive bayesian classifier for successful treatment of nonunions. J Bone Joint Surg Am 93:187-194. https://doi.org/10.2106/JBJS.I. 01649

38. Cebrián JL, Gallego P, Francés A et al (2010) Comparative study of the use of electromagnetic fields in patients with pseudoarthrosis of tibia treated by intramedullary nailing. Int Orthop 34:437-440. https://doi.org/10. 1007/s00264-009-0806-1

39. Zhai L, Ma X-L, Jiang C et al (2016) Human autologous mesenchymal stem cells with extracorporeal shock wave therapy for nonunion of long bones. Indian J Orthop 50:543-550. https://doi.org/10.4103/0019-5413. 189602

40. Stewart SK (2019) Fracture non-union: a review of clinical challenges and future research needs. Malays Orthop J 13:1-10. https://doi.org/10.5704/ MOJ.1907.001

41. Garcia P, Langer M, Raschke M (2015) The biological knife I-fracture healing and patient-dependent influencing factors. Z Orthop Unfall 153:433-440. https://doi.org/10.1055/s-0035-1546139

42. Calori GM, Albisetti W, Agus A et al (2007) Risk factors contributing to fracture non-unions. Injury 38(Suppl 2):S11-S18

43. Calori GM, Colombo M, Mazza EL et al (2014) Validation of the non-union scoring system in 300 long bone non-unions. Injury 45(Suppl 6):S93-S97. https://doi.org/10.1016/j.injury.2014.10.030

44. Patel RA, Wilson RF, Patel PA, Palmer RM (2013) The effect of smoking on bone healing: a systematic review. Bone Joint Res 2:102-111. https://doi. org/10.1302/2046-3758.26.2000142

45. Schaden W, Mittermayr R, Haffner N et al (2015) Extracorporeal shockwave therapy (ESWT) - first choice treatment of fracture non-unions? Int J Surg 24:179-183. https://doi.org/10.1016/j.ijsu.2015.10.003

46. Everding J, Stolberg-Stolberg J, Pützler J et al (2020) Extracorporal shock wave therapy for the treatment of arthrodesis non-unions. Arch Orthop Trauma Surg 32:45. https://doi.org/10.1007/s00402-020-03361-2

47. Wang FS, Yang KD, Chen RF et al (2002) Extracorporeal shock wave promotes growth and differentiation of bone-marrow stromal cells towards osteoprogenitors associated with induction of TGF-beta1. J Bone Joint Surg $\mathrm{Br}$ 84:457-461

48. Wang FS, Yang KD, Kuo Y-R et al (2003) Temporal and spatial expression of bone morphogenetic proteins in extracorporeal shock wave-promoted healing of segmental defect. Bone 32:387-396

49. Kubiak EN, Beebe MJ, North Ket al (2013) Early weight bearing after lower extremity fractures in adults. J Am Acad Orthop Surg 21:727-738. https:// doi.org/10.5435/JAAOS-21-12-727

50. Meadows TH, Bronk JT, Chao YS, Kelly PJ (1990) Effect of weight-bearing on healing of cortical defects in the canine tibia. J Bone Joint Surg Am $72: 1074-1080$

\section{Publisher's Note}

Springer Nature remains neutral with regard to jurisdictional claims in published maps and institutional affiliations. 